\title{
Influence du poids et de la densité de plantation des bulbes d'échalote sur les caractéristiques de la récolte et la prolificité des bulbes-fils
}

\author{
Joseph COHAT \\ avec la collaboration technique de Corentin Tromeur \\ I.N.R.A., Station d'Amélioration de la Pomme de terre et des Plantes à bulbes, F 29207 Landerneau
}

RÉSUMÉ

\begin{abstract}
Des bulbes d'échalote de Jersey (variété « Demi-longue ») de poids variable sont plantés à diverses densités. Le rendement augmente légèrement lorsque le poids planté par unité de surface augmente. La répartition de la récolte selon la grosseur des bulbes dépend du nombre de pousses et en conséquence de celui des bulbes formés à l'unité de surface. Ce nombre est fonction à la fois du poids de la semence et de la densité de plantation. La compétition plus ou moins forte entre pousses dans les cultures influence légèrement le rendement, mais surtout la prolificité des bulbes-fils quand ceux-ci sont utilisés pour une nouvelle plantation.
\end{abstract}

Mots clés additionnels : Allium cepa $L$. var. aggregatum, multiplication végétative, compétition, arrière effet.

Effect of weight and planting density of shallot bulbs on yield characteristics and the behaviour of daughter bulbs.

Shallot bulbs (Jersey variety "Demi-longue ») of various weights were planted at different densities. Yield increased slightly with weight of bulbs planted per unit area. The size distribution of harvested bulbs depended on the number of bulbs harvested per unit area, which in turn depended on both the weight of planted bulb and the planting density. The level of competition within the mother bulb crops slightly influenced yield and more clearly the rate of multiplication of daughter bulbs.

Additional key words : Allium cepa $L$. var aggregatum, vegetative propagation, competition, after effect.

\section{INTRODUCTION}

L'échalote est multipliée végétativement à partir de bulbes. Les plantations sont faites à des densités variables (de 10 à 25 bulbes au $\mathrm{m}^{2}$ ) et en utilisant des bulbes de différentes grosseurs (en général de 10 à $20 \mathrm{~g}$ ). Il n'existe pas de règles précises dans ce domaine, le choix des producteurs étant plus guidé par des données empiriques que par des faits expérimentaux bien établis. C'est pourquoi il nous a paru intéressant de mieux connaître l'iníluence du poids et de la densité de plantation des bulbes sur le rendement d'une culture d'échalote et les caractéristiques de la récolte en ce qui concerne en particulier le nombre, le calibre et la capacité de multiplication des bulbes-fils.

Une première étude (COHAT, 1982) a montré que le nombre de bulbes récoltés par touffe est en relation directe avec le poids du bulbe planté, du moins dans la gamme de poids étudiée $(7,5$ à $22,5 \mathrm{~g})$ et la densité de plantation utilisée ( 16 bulbes $\left./ \mathrm{m}^{2}\right)$. Si le rendement n'est que légèrement affecté, par contre la répartition des bulbes récoltés selon leur grosseur semble différer beaucoup selon le poids du bulbe planté. Depuis, de nouveaux essais faisant intervenir à la fois le poids de la semence et la densité de plantation ont été mis en place.

Les résultats obtenus sont présentés dans cette note et le choix de la densité de plantation et de la grosseur des semences à utiliser pour la production de l'échalote fait l'objet d'une discussion.

\section{MATÉRIEL ET MÉTHODES}

Dans un $1^{\text {er }}$ essai (A), des bulbes de poids moyen de 10,15 et $20 \mathrm{~g}$ ont été plantés aux 4 densités suivantes : $14,16,22$ et 29 bulbes au $\mathrm{m}^{2}$. Cet essai disposé en split-plot comportait des parcelles élémentaires de 
55 bulbes répétées 2 fois en 1983 et des parcelles de 70 bulbes répétées 4 fois en 1984. Les grandes parcelles étaient plantées aux mêmes densités. Pour des planches de $90 \mathrm{~cm}$ de large, séparées par des allées de $50 \mathrm{~cm}$, les diverses densités étaient obtenues par respectivement 4, 4, 5 et 6 lignes, avec des intervalles sur la ligne de $20,17,16$ et $15 \mathrm{~cm}$.

Dans un $2^{\mathrm{e}}$ essai (B), nous avons comparé 4 combinaisons poids de bulbe - densité de plantation. Chaque combinaison a été calculée de manière à obtenir le même nombre de bulbes-fils à l'unité de surface. Pour ce faire, compte tenu des essais antérieurs (COHAT, 1982), nous avons planté 4 lots de bulbes de poids moyen de 7,5 - 12,5 - 17,5 et $22,5 \mathrm{~g}$ aux densités suivantes : $29,22,16$ et 14 bulbes au $\mathrm{m}^{2}$. Compte tenu de leur taux présumé de multiplication (nombre de bulbes-fils produits) de 5,6 - 7,5 - 10,0 et 11,7, le nombre de bulbes-fils par $\mathrm{m}^{2}$ à attendre était de l'ordre de 160 . Ces divers traitements représentés par 55 à 60 bulbes par parcelle élémentaire étaient disposés en blocs à 4 répétitions.

Les plantations ont été faites début mars sur sol paillé de plastique noir. Les semences utilisées de la variété «Demi-longue " avaient été produites sur la station l'année précédente. La récolte a été effectuée fin juillet à début août ; elle a porté sur 40 à 50 touffes par traitement après élimination des plantes contiguës à 2 parcelles. Les bulbes ont été séchés, nettoyés et calibrés sur des grilles à lattes (écartements de 13, $19,25,28$ et $32 \mathrm{~mm}$ ), pesés et comptés.

Dans la récolte de chaque traitement, des bulbes de 10 et de $20 \mathrm{~g}$ ont été prélevés et replantés l'année suivante à la densité couramment utilisée de 16 bulbes au $\mathrm{m}^{2}$ (essai bloc à 4 répétitions, parcelle élémentaire de 24 bulbes). Les récoltes de ces essais sont analysées comme précédemment.

\section{RÉSULTATS}

\section{A. Influence du poids des semences et de la densité de plantation sur la récolte d'une culture}

L'effet de la densité de plantation et de la grosseur des semences se traduit au niveau de l'appareil végétatif par des différences importantes. Les bulbes de petit calibre, plantés à faible densité, donnent des plantes à feuilles vigoureuses, de couleur vert foncé, à gros collet et de maturité tardive ; les plantes issues de gros bulbes plantés à forte densité ont par contre des feuilles fines de couleur claire et mûrissent plus tôt. Les caractéristiques des plantes obtenues par les autres combinaisons densité-calibre se situent entre ces 2 extrêmes.

Le tableau 1 rassemble les résultats obtenus en 1983 et 1984 pour les 12 traitements étudiés en ce qui concerne le poids récolté par touffe et le rendement par ha.

Le rendement par touffe augmente avec le poids de la semence utilisée (par exemple en 1984, de 165 à $192 \mathrm{~g}$ lorsque le poids de la semence passe de 10 à $20 \mathrm{~g}$ ), il diminue lorsque la densité de plantation augmente (en 1984 de 214 à $131 \mathrm{~g}$ quand la densité de plantation varie de 14 à 29 bulbes au $\mathrm{m}^{2}$ ).

Le rendement par ha peut être plus ou moins affecté par ces 2 facteurs, le handicap apporté par l'utilisation d'une faible densité pouvant être en partie compensé par la plantation de bulbes de gros calibre. Les rendements les plus élevés sont toujours obtenus par la plantation de grosses semences à forte densité, c'est-à-dire avec le plus fort tonnage de semences plantées. Si l'effet calibre est significatif pour les 2 années, l'effet densité ne l'est qu'en 1984. Comme il n'existe pas d'interaction significative entre les 2 facteurs étudiés, il est possible d'établir une relation linéaire entre le rendement $\mathrm{y}$ et le poids $\mathrm{x}$ des bulbes plantés par ha pour chacune des 2 années. Cette relation n'est bien entendu valable que pour les valeurs de $\mathrm{x}$ comprises dans l'intervalle étudié, soit $1,4 \leqslant x \leqslant 5,8 \mathrm{t} / \mathrm{ha}$ et pour les conditions de chaque essai.

En 1983, elle se définit comme suit :

$$
\begin{array}{ll}
\mathrm{y}=1,92 \mathrm{x}+21,9 & \mathrm{r}=0,959 \\
\text { En } 1984: & \\
\mathrm{y}=3,00 \mathrm{x}+25,9 & \mathrm{r}=0,935 .
\end{array}
$$

L'effet du tonnage planté est donc plus important en 1984 qu'en 1983.

Le tableau 2 présente, pour les 12 traitements de 1983 , le nombre de bulbes récoltés par touffe, par $\mathrm{m}^{2}$ et le poids des bulbes de divers calibres dans la récolte totale.

Le nombre de bulbes-fils récoltés par touffe ne dépend que du calibre de la semence, quelle que soit la densité étudiée. Il est respectivement de 7,1 pour les semences de $10 \mathrm{~g}, 9,3$ pour celles de $15 \mathrm{~g}$ et 11,4 pour celles de $20 \mathrm{~g}$. Il en résulte que le nombre de bulbes obtenus par unité de surface augmente à la fois avec la densité et le poids de la semence, passant ainsi de 100 à $330 \mathrm{au} \mathrm{m}^{2}$ pour les 2 traitements extrêmes, soit un rapport de 1 à 3,3. Par contre, ce rapport ne se retrouve pas au niveau du rendement par ha qui passe seulement de 1 à 1,3 .

Il en résulte que le poids moyen des bulbes récoltés est d'autant plus faible que leur nombre augmente. Tout ceci conduit à des récoltes dans lesquelles les répartitions des bulbes selon leur calibre sont fort différentes. Si le nombre de bulbes formés est faible, comme par exemple dans le cas de plantations à faible densité de semences de petit calibre, la récolte est essentiellement composée de gros bulbes. On observe le contraire dans le cas de gros bulbes plantés à forte densité. Ceci est illustré par les courbes de la figure 1 donnant la répartition en poids des bulbes de calibre inférieur et supérieur à $28 \mathrm{~mm}$ dans la récolte en fonction du nombre de bulbes récoltés par unité de surface.

Il est particulièrement intéressant de noter que cette répartition ne dépend que du nombre de bulbes formés à l'unité de surface, quelle que soit la manière dont il a été obtenu, soit en agissant sur la densité de plantation, le poids des semences, ou sur les 2 à la fois.

Les traitements bulbes de $10 \mathrm{~g}$ plantés à $29 / \mathrm{m}^{2}$, de $15 \mathrm{~g}$ à $22 / \mathrm{m}^{2}$ et de $20 \mathrm{~g}$ à $16 / \mathrm{m}^{2}$ qui ont des peuplements de bulbes-fils voisins ont des récoltes de composition très proche. Ce fait est particulièrement vérifié par les résultats de l'essai B donnés dans les tableaux 3 et 4 . Nous avons ici comparé 4 combinaisons entre les 2 facteurs étudiés (poids des semences et densité de plantation) qui devaient en principe conduire à récolter le même nombre de bulbes à l'unité de surface. C'est 
TABLEAU 1

Influence du poids et de la densité de plantation de la semence sur le rendement.

Effect of bulb size and spacing on yield.

\begin{tabular}{|c|c|c|c|c|c|c|c|c|c|c|c|}
\hline & & \multicolumn{5}{|c|}{ Poids récolté par touffe $(\mathrm{g})$} & \multicolumn{5}{|c|}{ Rendement $\mathrm{t} / \mathrm{ha}$} \\
\hline \multirow{2}{*}{\multicolumn{2}{|c|}{$\begin{array}{l}\text { Calibre } \\
\text { (g) }\end{array}$}} & \multicolumn{5}{|c|}{ Densité $/ \mathrm{m}^{2}$} & \multicolumn{5}{|c|}{ Densité $/ \mathrm{m}^{2}$} \\
\hline & & 14 & 16 & 22 & 29 & $\bar{x}$ & 14 & 16 & 22 & 29 & $\bar{x}$ \\
\hline \multirow{5}{*}{$\stackrel{\mathscr{\Omega}}{\Omega}$} & 10 & 182 & 161 & 113 & 92 & 137 & 25,5 & 25,8 & 25,0 & 26,8 & $25,8 \mathrm{a}$ \\
\hline & 15 & 184 & 165 & 127 & 107 & 146 & 25,8 & 26,5 & 27,9 & 31,0 & 27,8 a b \\
\hline & 20 & 193 & 169 & 142 & 113 & 154 & 27,0 & 27,0 & 31,2 & 32,9 & $29,5 \mathrm{~b}$ \\
\hline & $\bar{x}$ & 187 & 165 & 127 & 104 & & 26,1 & 26,4 & 28,0 & 30,2 & \\
\hline & & & & & & & a & a & a & a & \\
\hline \multirow{5}{*}{$\stackrel{\square}{2}$} & 10 & 201 & 184 & 154 & 121 & 165 & 28,1 & 29,5 & 33,9 & 35,0 & $31,6 \mathrm{a}$ \\
\hline & 15 & 210 & 211 & 169 & 132 & 180 & 29,4 & 33,8 & 37,3 & 38,2 & $34,7 \mathrm{~b}$ \\
\hline & 20 & 230 & 217 & 179 & 141 & 192 & 32,2 & 34,7 & 39,4 & 41,1 & $36,8 \mathrm{c}$ \\
\hline & $\bar{x}$ & 214 & 204 & 167 & 131 & & 29,9 & 32,6 & 36,8 & 38,1 & \\
\hline & & & & & & & a & b & c & $c$ & \\
\hline
\end{tabular}

Les chiffres d'une colonne ou d'une ligne suivis d'une même lettre ne sont pas significativement différents au niveau 5 p. 100. (Méthode des distances multiples de DUNCAN.)

TABLEAU 2

Influence du poids et de la densité de plantation de la semence sur les caractéristiques de la récolte.

Effect of bulb size and spacing on yield characteristics.

\begin{tabular}{|c|c|c|c|c|c|c|c|c|}
\hline \multirow{2}{*}{ 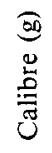 } & \multirow{2}{*}{ 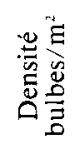 } & \multirow{2}{*}{$\begin{array}{l}\text { Nombre de bulbes } \\
\text { récoltés par } \mathrm{m}^{2} \\
\text { (par touffe) }\end{array}$} & \multicolumn{5}{|c|}{ Répartition de la récolte (t/ha) } & \multirow{2}{*}{$\begin{array}{c}\text { Nombre de bulbes de } \\
\text { calibre } 19 / 28 \mathrm{~mm} \\
\text { par } \mathrm{m}^{2}\end{array}$} \\
\hline & & & $13 / 19 \mathrm{~mm}$ & $19 / 25 \mathrm{~mm}$ & $25 / 28 \mathrm{~mm}$ & $28 / 32 \mathrm{~mm}$ & $>32 \mathrm{~mm}$ & \\
\hline \multirow{4}{*}{10} & 14 & $100(7,1)$ & 0,1 & 1,5 & 3,1 & 5,1 & 15,3 & 30 \\
\hline & 16 & $113(7,1)$ & 0,2 & 2,8 & 4,6 & 7,0 & 11,1 & 48 \\
\hline & 22 & $156(7,1)$ & 1,0 & 7,7 & 6,5 & 5,0 & 4,5 & 100 \\
\hline & 29 & $197(7,0)$ & 2,2 & 11,5 & 8,0 & 4,0 & 1,1 & 143 \\
\hline \multirow{4}{*}{15} & 14 & $133(9,5)$ & 0,3 & 3,9 & 5,4 & 7,2 & 8,8 & 64 \\
\hline & 16 & $146(9,1)$ & 0,8 & 5,8 & 5,8 & 7,1 & 6,9 & 78 \\
\hline & 22 & $204(9,3)$ & 2,2 & 11,4 & 8,4 & 4,7 & 1,1 & 145 \\
\hline & 29 & $275(9,5)$ & 4,6 & 17,4 & 7,1 & 1,5 & 0,3 & 188 \\
\hline \multirow{4}{*}{20} & 14 & $172(12,3)$ & 1,3 & 8,9 & 7,8 & 5,4 & 3,5 & 117 \\
\hline & 16 & $185(11,6)$ & 1,9 & 10,0 & 7,3 & 4,9 & 2,4 & 125 \\
\hline & 22 & $264(12,0)$ & 4,0 & 18,1 & 6,2 & 2,5 & 0,3 & 187 \\
\hline & 29 & $330(11,4)$ & 7,9 & 19,1 & 4,6 & 1,0 & 0,2 & 194 \\
\hline
\end{tabular}

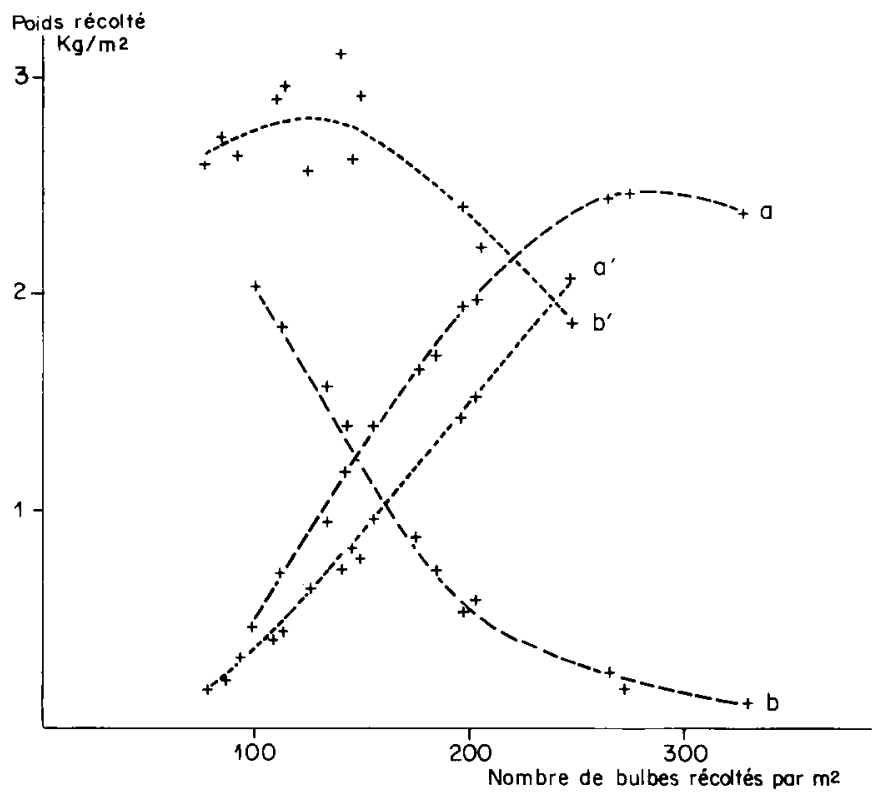

Figure 1

Répartition de la récolte en 2 calibres en fonction du nombre de bulbes récoltés par unité de surface.

Grading of yield according to the number of harvested bulbs per unit area.

a, $a^{\prime}$ : poids de bulbes petits et moyens $(19 \mathrm{~mm}<\varnothing<28 \mathrm{~mm})$ récoltés par $m^{2}$ en 1983 (a) et $1984\left(a^{\prime}\right)$.

$b, b^{\prime}$ : poids de gros bulbes $(\varnothing>28 \mathrm{~mm})$ récoltés par $\mathrm{m}^{2}$ en 1983 (b) et $1984\left(b^{\prime}\right)$. 
TABLEAU 3

Influence du poids et de la densité de plantation de la semence sur les caractéristiques de la récolte.

Effect of bulb size and planting density on yield characteristics.

\begin{tabular}{ccccc}
\hline $\begin{array}{c}\text { Calibre } \\
(\mathrm{g})\end{array}$ & $\begin{array}{c}\text { Nombre de bulbes } \\
\text { plantés } / \mathrm{m}^{2}\end{array}$ & $\begin{array}{c}\text { Taux de } \\
\text { multiplication }\end{array}$ & $\begin{array}{c}\text { Nombre de bulbes } \\
\text { récoltés } / \mathrm{m}^{2}\end{array}$ & $\begin{array}{c}\text { Poids récolté } \\
\text { par touffe }(\mathrm{g})\end{array}$ \\
\hline 7,5 & 29 & 5,6 & 163 & 102 \\
12,5 & 22 & 8,0 & 177 & 145 \\
17,5 & 16 & 10,6 & 170 & 192 \\
22,5 & 14 & 12,4 & 174 & 31,9 \\
$\mathrm{t}$ & & & 212 & 29,7 \\
\hline
\end{tabular}

TABLEAU 4

Influence du poids et de la densité de plantation de la semence sur la répartition de la récolte en différents calibres (t/ha). Effect of bulb size and planting density on the size distribution of harvested bulbs.

\begin{tabular}{|c|c|c|c|c|c|c|}
\hline \multirow{2}{*}{$\begin{array}{l}\text { Calibre } \\
(\mathrm{g})\end{array}$} & \multirow{2}{*}{$\begin{array}{c}\text { Densité } \\
\text { bulbes } / \mathrm{m}^{2}\end{array}$} & \multicolumn{5}{|c|}{ Répartition de la récolte ( $\mathrm{t} / \mathrm{ha}$ ) } \\
\hline & & $13 / 19 \mathrm{~mm}$ & $19 / 25 \mathrm{~mm}$ & $25 / 28 \mathrm{~mm}$ & $28 / 32 \mathrm{~mm}$ & $>32 \mathrm{~mm}$ \\
\hline 7,5 & 29 & 0,3 & 5,8 & 6,8 & 8,2 & 8,5 \\
\hline 12,5 & 22 & 0,5 & 7,0 & 9,0 & 8,5 & 6,8 \\
\hline 17,5 & 16 & 0,7 & 6,5 & 7,2 & 8,6 & 7,6 \\
\hline 22,5 & 14 & 0,9 & 6,4 & 7,3 & 8,1 & 6,9 \\
\hline
\end{tabular}

ce qui a été presque obtenu en précisant cependant que ce nombre est légèrement supérieur à celui attendu. Les rendements des 4 traitements sont sensiblement du même ordre et la répartition des bulbes récoltés selon leur calibre est relativement homogène (tabl. 4). Il faut aussi ajouter que, dans cet essai, aucune différence dans les caractéristiques de l'appareil végétatif des plantes des 4 traitements n'a été notée, tant pour la vigueur que pour la coloration et la précocité de maturation.

En conclusion, le niveau et la composition de la récolte d'une culture d'échalote paraît donc dépendre du nombre de bulbes-fils formés par unité de surface, c'est-à-dire, en d'autres termes, de leur densité de peuplement. Il est possible de le modifier en agissant sur 2 facteurs, grosseur des semences et densité de plantation.

\section{B. Influence des conditions de plantation d'une cul- ture sur le comportement des bulbes-fils}

Le tableau 5 donne les rendements obtenus en plantant des bulbes prélevés dans les récoltes des 12 traitements de l'essai A de 1983. Bien que faible, l'effet des traitements réalisés en 1983 persiste encore en 1984 de manière significative. En particulier, les bulbes issus des cultures présentant les nombres les plus faibles de bulbes-fils ont un potentiel de rendement plus élevé.

Le tableau 6 montre que les taux de multiplication des bulbes des divers traitements sont significativement différents. En confrontant le classement des 12 traitements réalisés en 1983 selon l'ordre croissant de la densité de peuplement des bulbes-fils à la récolte à celui de la moyenne des taux de multiplication en 1984 des bulbes de 10 et $20 \mathrm{~g}$, il est possible de calculer le coefficient de corrélation des rangs de SPEAR-

\section{TABLEAU 5}

Influence du poids et de la densité de plantation des bulbes-mères en 1983 sur le rendement des bulbes-fils en 1984 (moyenne des rendements obtenus avec des semences de 10 et $20 \mathrm{~g}$ en $\mathrm{t} / \mathrm{ha}$ ). Effect of size and spacing of mother bulbs on the yield of daughter bulbs.

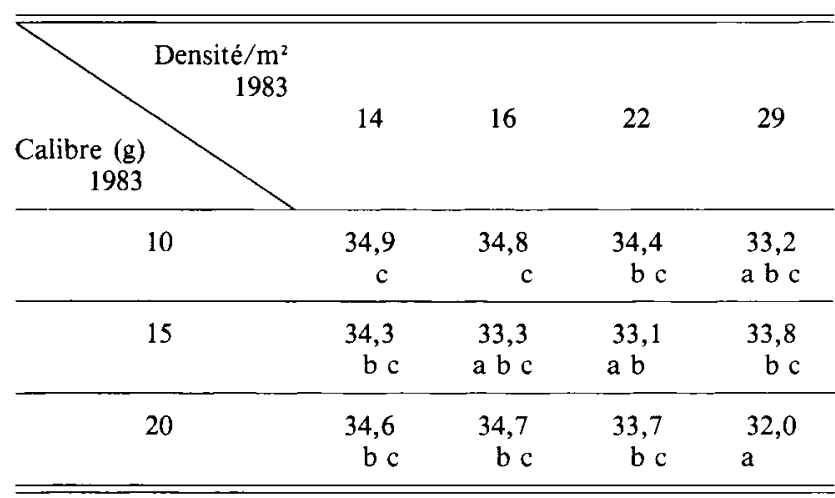

Les chiffres suivis d'une même lettre ne sont pas significativement différents au niveau 5 p. 100. (Méthode des distances multiples de DUNCAN.)

MANN (SNEDECOR \& COCHRAN, 1957). Celui-ci est égal à $-0,923$ et est significatif au seuil de 1 p. 100 . Ceci veut dire que la densité de peuplement de bulbesfils d'une culture influence la prolificité des bulbes récoltés, les cultures possédant les peuplements les plus élevés produisent les bulbes-fils les moins prolifiques et réciproquement.

Le tableau 7 rassemble les résultats de la replantation en 1984 de bulbes de 10 et $20 \mathrm{~g}$ prélevés dans l'essai $\mathrm{B}$ où les récoltes des divers traitements étaient très proches les unes des autres quant à leur niveau et leur composition. Les rendements obtenus en 1984 
TABLEAU 6

Influence du poids et de la densité de plantation des bulbes-mères en 1983 sur la prolificité des bulbes-fils en 1984. Effect of size and spacing of mother bulbs on the rate of multiplication of daughter bulbs.

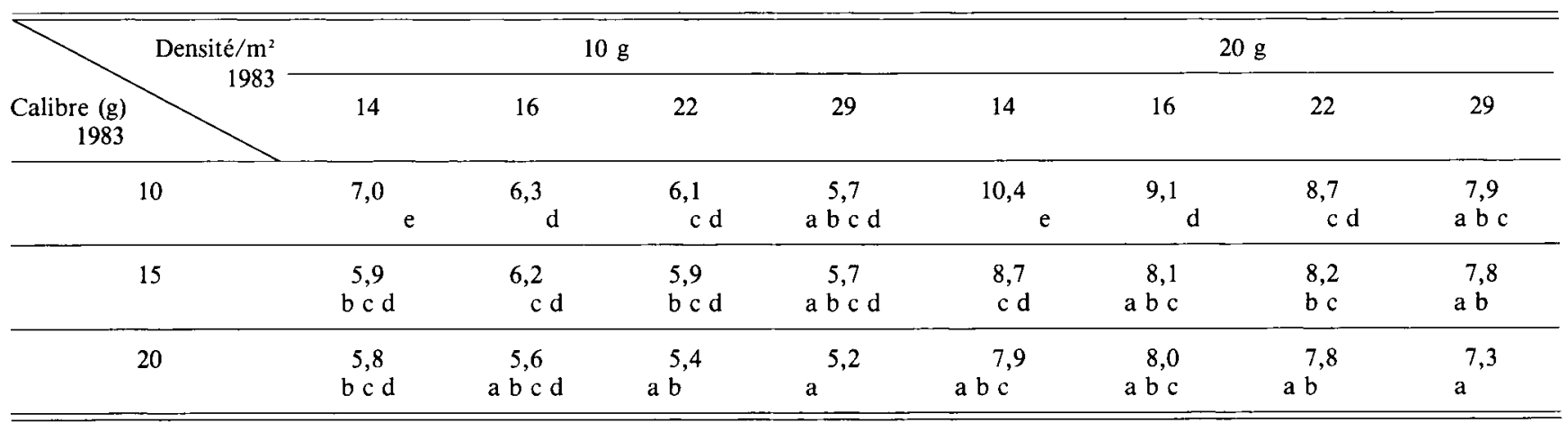

Les chiffres suivis d'une même lettre ne sont pas significativement différents au niveau 5 p. 100. (Méthode des distances multiples de DuNCAN.)

TABLEAU 7

Influence du poids et de la densité de plantation des bulbes-mères sur le comportement des bulbes-fils.

Effect of size and spacing of mother bulbs on the behaviour of daughter bulbs.

\begin{tabular}{rcccccc}
\hline \hline \multirow{2}{*}{ Issu de } & \multicolumn{2}{c}{ Rendement $\mathrm{t} / \mathrm{ha}$} & \multicolumn{4}{c}{$\begin{array}{c}\text { Taux de } \\
\text { multiplication }\end{array}$} \\
\cline { 2 - 7 } & $10 \mathrm{~g}$ & $20 \mathrm{~g}$ & & $10 \mathrm{~g}$ & $20 \mathrm{~g}$ \\
\hline $7,5 \mathrm{~g}$ à $29 / \mathrm{m}^{2}$ & $30,1 \mathrm{a}$ & $32,7 \mathrm{a}$ & $5,9 \mathrm{a}$ & $9,5 \mathrm{a}$ \\
$12,5 \mathrm{~g}$ à $22 / \mathrm{m}^{2}$ & $31,1 \mathrm{a}$ & $32,9 \mathrm{a}$ & 5,6 & $\mathrm{~b}$ & $9,1 \mathrm{a} \mathrm{b}$ \\
$17,5 \mathrm{~g}$ à $16 / \mathrm{m}^{2}$ & $32,0 \mathrm{a}$ & $33,8 \mathrm{a}$ & 5,5 & $\mathrm{~b} \mathrm{c}$ & 8,8 & $\mathrm{~b}$ \\
$22,5 \mathrm{~g}$ à $14 / \mathrm{m}^{2}$ & $32,5 \mathrm{a}$ & $33,7 \mathrm{a}$ & 5,3 & $\mathrm{c}$ & 8,1 & $\mathrm{c}$ \\
\hline \hline
\end{tabular}

sont du même ordre, ce qui est conforme aux conclusions émises précédemment, le peuplement de bulbesfils étant le même en 1983 pour les 4 traitements étudiés.

Toutefois, il a été observé que la prolificité d'un bulbe pouvait aussi être influencée par le poids du bulbe-mère. En effet, les bulbes obtenus en 1983 dans cet essai $\mathrm{B}$ à partir de petites semences sont plus prolifiques que ceux obtenus à partir de grosses semences.

\section{DISCUSSION ET CONCLUSION}

Le poids et la densité de plantation des semences utilisées influencent à la fois le niveau et la composition d'une récolte d'échalote et la prolificité des bulbes-fils quand ceux-ci sont utilisés pour une nouvelle culture.

L'analyse de la structure anatomique du bulbe d'échalote et de ses caractéristiques physiologiques peut permettre de mieux comprendre le mécanisme d'action de ces 2 facteurs.

Un bulbe d'échalote est composé d'un plateau sur lequel sont insérés des bases de feuilles charnues ou non, des écailles et des points végétatifs. Après la plantation, chaque bourgeon forme de 5 à 7 feuilles dont 2 à 5 vont s'épaissir à la base, puis se divise pour former 2 nouveaux points végétatifs qui vont former 2 à 3 feuilles ou écailles avant de se diviser à leur tour et ainsi de suite. Le nombre de bourgeons d'un bulbe semble être déterminé dès la récolte. Les bourgeons présents chez le bulbe-mère au moment de la plantation paraissent se développer indépendamment les uns des autres. Contrairement à ce qui se passe chez d'autres plantes à bulbes, comme la tulipe ou l'iris (REES, 1972), il ne semble pas exister de phénomène de prééminence ou de dominance entre les bourgeons d'un même bulbe qui se développent tous simultanément pour donner des pousses dont la base de chacune formera un bulbe-fils. L'unité de population à considérer n'est pas le bulbe-mère et la touffe à laquelle il donne naissance, mais la pousse issue de chaque bourgeon. Plus le nombre de pousses se développant à l'unité de surface sera grand, plus le niveau de compétition entre pousses sera élevé, ce qui limitera le grossissement individuel de chaque bulbe-fils. C'est ce qui a été observé dans le cas d'une plantation de gros bulbes à forte densité. Réciproquement, l'intensité de la compétition sera moins forte quand le nombre de pousses diminue, ce qui favorisera le grossissement des bulbes-fils. C'est le cas d'une plantation de petits bulbes à faible densité.

Les rendements les plus élevés ont été obtenus dans nos essais avec les nombres de bulbes-fils les plus grands. Cependant, il est fort probable que des peuplements plus denses entraînant une plus forte compétition entre pousses conduiraient à une diminution du rendement. De tels peuplements n'ont pas été expérimentés, car leur intérêt pratique est limité pour diverses raisons : valeur élevée de la semence à planter, travail important de plantation, risques sanitaires accrus et poids moyen des bulbes récoltés insuffisant.

L'influence du poids de la semence et de la densité de plantation sur la prolificité des bulbes-fils est plus difficile à expliquer. Il est possible de concevoir ici que des bulbes de même poids obtenus dans des conditions différentes de compétition possèdent des structures différentes: nombre différent de bourgeons, nombre plus ou moins grand d'écailles entre chaque division du bourgeon, écailles plus ou moins épaisses. Seule l'étude précise de la structure de bulbes provenant des différents traitements apporterait une réponse à ces questions. 
Toutefois dans des conditions très proches de compétition (essai B), il subsiste des différences de prolificité entre bulbes-fils des divers traitements, ces différences pouvant être imputées au poids de la semence. On peut tenter d'expliquer l'effet de ce facteur, soit par le nombre différent de bourgeons des bulbes plantés entraînant une intensité variable de compétition à l'intérieur de la touffe, soit en considérant la qualité des bourgeons de bulbes de différents poids. Cette qualité serait liée au rapport masse de réserves/nombre de bourgeons, dont la valeur est plus élevée pour les gros bulbes. A partir du tableau 3, il est possible de la calculer ; elle est respectivement de 1,34-1,56-1,65 et 1,81 pour des bulbes de $7,5-12,5-17,5$ et $22,5 \mathrm{~g}$. L'étude des bulbes-fils issus de bulbes dont on a enlevé une partie des réserves sans modifier le nombre de bourgeons devrait permettre de vérifier cette hypothèse.

L'influence des conditions de production sur la prolificité ultérieure des bulbes-fils introduit une notion d'interdépendance entre générations successives d'une descendance clonale. Cette notion n'est pas nouvelle ; elle a été aussi mise en évidence chez la pomme de terre (PERENNEC \& MADEC, 1979), la tulipe (LE NARD, 1983) et semble être une règle assez générale chez les plantes se multipliant végétativement par un organe de réserve comme un tubercule, un bulbe, etc...

Elle constitue un élément important à considérer lors de la mise en place d'une expérimentation sur échalote. Il paraît ainsi indispensable de réaliser au préalable une multiplication des semences sous les mêmes conditions à partir de matériel de calibre homogène.

D'un point de vue pratique, les résultats des expériences entreprises apportent d'utiles enseignements au producteur d'échalote pour l'aider à déterminer les meilleures conditions de plantation.

Au moment de la plantation, il est important de penser à la fois au calibre des semences et à la densité de plantation, afin d'obtenir un peuplement optimum de bourgeons à l'unité de surface, bourgeons qui deviendront plus tard des bulbes-fils. Ceci peut être obtenu en jouant sur l'un ou l'autre de ces 2 facteurs.

Le peuplement optimum sera choisi en fonction de la capacité de production du lieu et des objectifs recherchés pour la grosseur des bulbes récoltés. Ainsi pour une culture destinée à la production de bulbes de semence, le producteur cherchera avant tout le plus grand nombre de bulbes de calibre moyen $(10$ à $20 \mathrm{~g})$ et de bonne qualité. Pour atteindre cet objectif, un peuplement dense de bourgeons doit être réalisé. Pour un rendement espéré de $25-30 \mathrm{t} / \mathrm{ha}$, une densité d'au moins 200 bulbes-fils au $\mathrm{m}^{2}$ doit être atteinte et une combinaison gros bulbe-densité moyenne ou basse de plantation doit de préférence être retenue, du moins pour les conditions de culture du Nord-Finistère.

Reçu le 10 juin 1985. Accepté le 26 août 1985.

\section{RÉFÉRENCES BIBLIOGRAPHIQUES}

Cohat J., 1982. Influence du calibre des bulbes de semence d'échalote sur leur taux de multiplication et leur rendement. Pépiniéristes, Horticulteurs, Maraîchers, $\mathrm{n}^{\circ}$ 231, 21-24.

Le Nard M., 1983. Physiology and storage of bulbs : concepts and nature of dormancy in bulbs in "Post-harvest physiology and crop preservation "). NATO Advanced Study Institutes Series ; Series A : Life Science, Vol. 46, 191-230. Ed. by M. Lieberman, Plenum Press, New York.
Perennec P., Madec P., 1979. Age physiologique du plant de pomme de terre. Incidence sur la germination et répercussions sur le comportement des plantes. Potato Res., 23, 183-199.

Rees A. R., 1972. The growth of bulbs. Academic Press ; London, New York.

Snedecor G. W., Cochran W. G., 1957. Méthodes statistiques (traduction française). Acta, Paris. 